\title{
LAS CLAVES DEL CONFLICTO ENTRE RUSIA Y OCCIDENTE DESPUÉS DE GRIMEA Y EL CONFLICTO CON UCRANIA
}

Ana Teresa Gutiérrez del Cid

\section{INTRODUCGIÓN}

$\mathrm{Al}$ inicio de 2013 se observaba un proceso consistente en un amplio giro geopolítico en Europa, que empezó con el reposicionamiento de Rusia como potencia regional y con el debilitamiento de la presencia de Estados Unidos en este continente, porque se hallaba inmerso en las intervenciones militares en Afganistán e Irak desde principios del decenio del 2000. Este giro inició aproximadamente en 2005. Además, Alemania se había acercado a Rusia, a la vez se había distanciado de Estados Unidos, en cuanto a sus nexos comerciales. Por estos cambios en la correlación geopolítica de fuerzas, surgieron nuevas tendencias de alineamiento de los países europeos. Así, el corredor europeo oriental que comprende los Estados Bálticos, Polonia, la República Checa, Eslovaquia, Hungría, Rumania y Bulgaria, denominado corredor Intermarium, emergió como un área de contención entre Rusia y Estados Unidos. Esto significa que la línea de batalla que dividía Europa entre los dos bloques de la Guerra Fría se movió hacia el Este y los países a lo largo de esta línea son aliados de Occidente. ${ }^{1}$

Rusia, a su vez, ha venido reposicionándose como gran potencia energética del siglo xxi y ha desarrollado una red de oleoductos y gasoductos que podría convertirla en el gran árbitro del

1 "Europe: A Shifting battleground, Part 1", Stratfor, 7 de junio de 2011, en www.stratfor.org 
suministro de petróleo y gas para Europa y los grandes centros económicos y demográficos del Asia Oriental (China, India, Japón, Corea del Sur). Aunque el auge de la extracción del gas shale en Estados Unidos y la baja de los precios del petróleo desde 2014 han afectado tanto a la economía rusa, como en la posibilidad de que Rusia tenga el monopolio de la distribución del petróleo y del gas en el mundo.

Esta nueva estrategia de Rusia ha cambiado la geopolítica tradicional de Europa durante la Guerra Fría, acercándola a Alemania por el consumo alemán de energéticos rusos, hecho que es contrario a las recomendaciones del padre de la visión geopolítica británica, Sir Halford Mackinder, que aconsejaba a las potencias marítimas la estrategia de separar a Alemania de Rusia.

Cada vez es más evidente, por tanto, que el orden unipolar del poder mundial cede espacio al multipolar. Este proceso, sin embargo, no puede transcurrir sin conflictos, ya que la aparición de nuevos actores internacionales quita poderío a las potencias ya establecidas acostumbradas a un liderazgo sin condiciones. Sin embargo, al inicio de su primer periodo presidencial, Barack Obama reconoció esta nueva realidad mundial e intentó articular una nueva política exterior para con Rusia, denominada reset, que fracasó por las presiones de los republicanos más renuentes a aceptar esta nueva realidad.

En esta transformación geopolítica se inscriben los acontecimientos que hoy atestiguamos en Ucrania, donde por un golpe de Estado, de manera muy violenta y aliándose con los nacionalistas ucranianos que poseen una ideología racista, antirrusa y antisemita, semejantes a la del nazismo alemán de Hitler, ${ }^{2}$ Occidente

${ }^{2}$ Los actuales grupos de nacionalistas ucranianos, denominados "fracción de derecha”, rinden pleitesía al ideólogo Suskievich, muy cercano a la ideología del nacional socialismo alemán de Hitler, y a Stepan Bandera, ejecutor del acercamiento de estos grupos en 1941, fecha en que la Alemania nazi atacó a la Unión Soviética. Al respecto, véase Daniel Lazare, "Who was Stepan Bandera?", Jacobin, 24 de septiembre de 2015, en https://www.jacobinmag.com/2015/09/stepanbandera-nationalist-euromaidan-right-sector/ y Grzegorz Rossoliński-Liebe, Stepan Bandera: The Life and Afterlife of a Ukrainian Nationalist, Fascism, Genocide, and Cult, Berlín, ibidem Press, 2014. 
colocó en el poder a un gobierno antirruso, que fracturó la constitucionalidad del poder presidencial en Ucrania, ya que Víctor Yanukóvich, presidente derrocado, fue elegido legalmente. Esta acción fue un acto desesperado ante la negativa del presidente Yanukóvich de firmar el Acuerdo de Asociación Oriental en Vilnius, en noviembre de 2013.

En el presente trabajo se analiza cómo el conflicto en Ucrania ha cambiado el proceso susodicho y ha enfrentado a los líderes de la Unión Europea (UE) y a Rusia, logrando volver al modelo geopolítico de la Guerra Fría que separó a la Europa occidental de la entonces Unión Soviética, por la formación de nuevas alianzas militares en Europa, la reincorporación de Crimea por parte de Rusia y la imposición de sanciones como respuesta de Europa a esta acción.

El Regreso de la estrategia de Mackinder

Si se analiza la situación mundial con detenimiento, hay tres sucesos muy importantes que han dado un giro al unipolarismo estadounidense: Edward Joseph Snowden, que no se devolvió a Estados Unidos, ya que no hay entre Rusia y este país una ley de extradición; el freno de un posible ataque de la Organización del Tratado del Atlántico Norte (оTAN) a Siria en 2013, logro de la gestión diplomática del presidente Vladimir Putin; el acuerdo entre Irán y Estados Unidos, que parecía imposible hace poco. Todos éstos son triunfos de la diplomacia rusa y empiezan a mostrar que el poder mundial ya no es unipolar y se transita a un nuevo orden mundial multipolar, situación que a su vez se refuerza con el surgimiento de los países BRICS, a los que Rusia pertenece.

Esta transformación del poder mundial, de unipolar a multipolar, se ha conceptualizado en el apartado "El mundo contemporáneo y la política exterior rusa” de la Concepción de política exterior de la federación rusa. La fundamental característica de la actual etapa de desarrollo internacional son los profundos cambios en el plano geopolítico, cuyo potente catalizador fue la crisis económico-financiera global. Las relaciones internacionales atraviesan por 
un periodo de transición, cuya esencia constituye la formación de un sistema internacional policéntrico. Este proceso transcurre con dificultad, acompañándose del aumento de turbulencia en el desarrollo económico y político en el mundo y la región. Las relaciones internacionales continúan complicándose y su desarrollo se vuelve cada vez más difícil de predecir. ${ }^{3}$

La proyección de esta nueva fuerza de la diplomacia rusa, que le ha hecho volver a tener un papel principal en los acontecimientos mundiales, es también un proceso preocupante para Estados Unidos. Occidente siempre ha tenido a Rusia por un gran país atemorizador. La base teórica de esta visión occidental se ha formado sobre todo a partir del pensamiento político y geopolítico anglosajón. Uno de los teóricos más destacados en este aspecto es Sir Halford Mackinder. ${ }^{4}$ La esencia de esta concepción y de los pioneros de la geopolítica occidental cercanos a él consiste en que todas las potencias del planeta pueden dividirse en marítimas y continentales. En esta visión, Eurasia posee un significado clave y se la denomina "isla-mundo", un espacio vital de la geopolítica mundial. El centro de este territorio lleva el nombre de heartland ('corazón de la tierra'), cuya localización se contrapone al rimland, extensión policéntrica en forma de mosaico. El heartland está representado por Rusia y constituye un monolito encuadrado interna y externamente por un territorio denominado "media luna". 5 La media luna interna son los países a lo largo del perímetro marítimo de Eurasia, del Báltico al Pacífico. La media luna externa está formada sobre todo por los países anglosajones: Estados Unidos,

${ }^{3}$ Концепция внешней политики Российской Федерации (Утверждена Презид ентом Российской Федерации В.В.Путиным 12 февраля 2013 г) (“Concepción de política exterior de la federación rusa, ratificada por el presidente de la federación rusa el 12 de febrero de 2013”), Совет Безопасности Российской Федерации (Consejo de Seguridad de la Federación Rusa), 2013, en http:/ /www.scrf.gov.ru/ documents $/ 2 / 25 . h$ tml

${ }^{4}$ Agustín Saavedra Weise, "Los 150 años de Sir Halford J. Mackinder”, Centro de Estudios Internacionales para el Desarrollo, Buenos Aires, Argentina, 21 de febrero de 2011, en http://www.ceid.edu.ar/biblioteca/2011/agustin_saavedra_ weise_los_150_de_sir_halford_j.pdf

${ }^{5}$ H. J. Mackinder, "The Geographical Pivot of History", en Democratic Ideals and Reality, Washington DC, National Defense University Press, 1996, pp. 175-194. 
Canadá y Gran Bretaña. Como sabemos, la argumentación más importante de Mackinder consiste en que quien domine Europa Oriental controlará el heartland, la isla mundo, y quien la posea someterá al mundo.

Hoy esta fórmula geopolítica no es tan representativa, ya que la escena internacional ha cambiado mucho desde las afirmaciones de Mackinder, pero aún conserva la esencia del pensamiento geopolítico occidental, que sigue percibiendo a Rusia como una amenaza. ${ }^{6} \mathrm{Y}$ es que es difícil no considerar el enorme territorio ruso como un peligro. La percepción de éste ha contribuido a definir la historia contemporánea. Por ejemplo, la participación de Estados Unidos en los asuntos europeos a lo largo del siglo xx, la creación del espacio euroatlántico y finalmente la creación de la Unión Europea (UE) pueden interpretarse como resultado de la aspiración de las potencias marítimas a equilibrar la vulnerabilidad de Europa con la masa terrestre oriental y euroasiática. Para tal fin, la geopolítica occidental, desde siempre, ha tenido la aspiración de desintegrar, ahí donde sea posible, el monolito eurasiático. En un principio se esforzó en impedir que Rusia tuviera aliados en Europa Occidental. Éste fue el propósito de la política y de las exitosas acciones de los anglosajones en los siglos xix y xx. También se logró separar después a Europa del Este de Rusia a finales del siglo xx y como último objetivo se ha pretendido lograr su desintegración misma y convertirla en un territorio poroso de regiones autónomas. ${ }^{7}$

La obra de Zbigniew Brzezinski, El gran tablero mundial. La supremacía estadounidense..., refleja los postulados fundamentales de la geopolítica occidental contemporánea. En este trabajo se encuentra el diseño de contención bajo control estadounidense de los grandes centros de poder en el litoral de Eurasia, después de la Guerra Fría: China, Irán, Turquía y los países de la uE. Para lograr

${ }^{6}$ Véase F. William Engdahl, "Ukraine and a Tectonic Shift in Heartland Power", 22 de marzo de 2010, en //www.engdahl.oilgeopolitics.net/print/Ukraine\%20 and \%20a\%20Tectonic\% 20Shift\%20in\%20Heartland\%20Power.pdf

${ }^{7}$ Cf. Zbigniew Brzezinski, "A Geostrategy for Eurasia”, Foreign Affairs, vol. 76, núm. 5, 1997, en https://www.foreignaffairs.com/articles/asia/1997-09-01/geostrategy-eurasia 
este fin, se plantea ampliar la influencia estadounidense en los territorios postsoviéticos y avanzar en el debilitamiento de Rusia.

Como en los trabajos de Mackinder, Eurasia se concibe otra vez como un espacio geopolítico, cuyo dominio abre la posibilidad de controlar el mundo, ya que después de la desintegración de la Unión Soviética hubo, en efecto, un cambio tectónico en la geopolítica mundial, "pues Estados Unidos por primera vez logró constituirse en un árbitro de las potencias euroasiáticas con presencia en la región y llegó a ser la mayor potencia mundial". 8

\section{EL FRACASO DEL RESET Y LA NUEVA REALIDAD DEL INTERMARIUM}

El abandono del reset (la estrategia para con Rusia que concibió el gobierno de Barack Obama en su primer periodo presidencial, movido por una estrategia belicista en Europa) coincide con las aspiraciones de los países del denominado Intermarium, término tomado del líder polaco Josef Pilsudsky, quien vivió en el periodo entre las dos guerras mundiales y que consideraba que Alemania y la Unión de Repúblicas Socialistas Soviéticas (URSs) no serían permanentemente débiles. Su propuesta fue una alianza que fortaleciera la región que comprende desde el Mar Báltico hasta el Mar Negro, incluyendo a los países al occidente de los Cárpatos. Hoy, este término en la geopolítica es útil como una manera de agrupar países colindantes con la esfera de influencia de Rusia y que están cautelosos de la nueva relación de Berlín con Moscú. Éstos son: Estados Bálticos, República Checa, Eslovaquia, Hungría, Rumania y Bulgaria, y puede incluirse a Suecia y Finlandia, ya que ambos países se muestran también cautelosos con respecto de Rusia y tienen interés en mantener a las Repúblicas Bálticas independientes de Moscú, porque consideran la región Báltica como su esfera de influencia y son los líderes del Grupo Nórdico.

En conclusión, el grupo denominado Intermarium intenta contener a Moscú y considera que hoy no podría apoyarse sólo en

${ }^{8}$ Véase Z. Brzezinski, El gran tablero mundial. La supremacía estadounidense y sus imperativos geoestratégicos, Barcelona, Paidós, 1998. 
Alemania para hacerlo. Además, este grupo considera también que el involucramiento de Estados Unidos en Medio Oriente, Asia Central y el norte de África, ha convertido a Europa del Este en segunda prioridad para Estados Unidos. Esta preocupación se evidenció en la decisión de Washington de alterar sus planes sobre el proyecto denominado Defensa Antimisil (DAM) en septiembre de 2009 (estrategia del reset de Obama), a cambio de concesiones rusas en Asia Central y en Afganistán, que consistían en lograr la aprobación rusa de sanciones contra Irán en el Consejo de Seguridad de las Naciones Unidas (ONU) y la concesión de una ruta a través del territorio ruso para suministrar los pertrechos de guerra estadounidenses para su ocupación de Afganistán.

A pesar de que actualmente el presidente estadounidense, Barack Obama, ha retomado el DAM, esta negociación entre Washington y Moscú mostró al Intermarium que los Estados Unidos no dudarían en priorizar la zona de Asia Central y Medio Oriente sobre una estrategia de largo plazo en la Europa del Este.

Estos países están respondiendo a la situación con dos estrategias principales. La primera es mantener el compromiso de Estados Unidos el mayor tiempo posible; la segunda, crear alianzas políticas y militares regionales, independientes de la OTAN, que puedan servir como alternativas a la presencia de Estados Unidos en la región, presencia que preferirían estos países. Sin embargo, el problema es que Estados Unidos, como ya se anotó, estaba inmerso en dos guerras en el Asia Central y Medio Oriente, y aunque está tratando de salir de Irak y Afganistán, aún está comprometido.

Por tanto, los países del Intermarium están formando dos alianzas regionales para crear coaliciones con otros países que también están preocupados con el reposicionamiento ruso: el Grupo Visegrad o V4, que incluye a Polonia, la República Checa, Eslovaquia y Hungría y el Grupo Nórdico Báltico. Estos dos grupos no tienen mucha relación entre sí, especialmente el Nórdico Báltico del Norte que incluye también al Reino Unido y a veces a Irlanda. El Nórdico Báltico se integró a principios de 2011 y consta de las tres repúblicas bálticas de la extinta Unión Soviética: Lituania, Estonia y Letonia, Noruega, que pertenecen a la OTAN, 
Suecia y Finlandia, que cooperan con esta alianza, sin pertenecer a ella. El Grupo de Visegrad decidió formar un batallón en mayo de 2011, bajo el mando polaco, para estar en funciones en 2016. Las capacidades de este grupo de batalla aún tienen que determinarse, pero la decisión muestra muy claramente que el V4 está formado por un grupo político que evolucionará a un grupo que establece como prioridad de su misión la formación de fuerzas militares.

Según George Friedman, la decisión del V4, a principios de 2011, es muy importante para Europa. ${ }^{9}$ El 12 de mayo de 2011 anunció la formación de un grupo de batalla bajo el mando de Polonia. Este grupo estaría listo en 2016 como una fuerza independiente y no sería parte del Comando de la otan. Por lo demás, en 2013 los cuatro países del V4 iniciaron ejercicios militares conjuntos, bajo los auspicios de la Fuerza de Respuesta de la otan. Estos países temen el fortalecimiento actual de Rusia y además la atracción con Europa se ha desgastado. Así, los países nórdicos comparten la suspicacia ante Rusia. Noruega también teme la actividad de Rusia en el Mar de Barents. Estos países y el Reino Unido estaban preocupados por el surgimiento de la relación ruso-germana, como ya se anotó.

Los países nórdico-bálticos tienen ya un grupo de batalla creado desde 2008, bajo el formato del grupo de batalla de la uE. Sus miembros actuales son: Suecia, Finlandia, Noruega, Estonia e Irlanda, si bien Lituania se unió en 2014. El propósito del Grupo Nórdico-Báltico es aumentar el componente militar, más allá del batallón que hay.

El Báltico Nórdico se reunió con el gobierno del Reino Unido en enero de 2011, para constituir un nuevo grupo cuyo objetivo estratégico fundamental fuese ampliar las relaciones con los estados miembros generalmente escépticos del liderazgo francogermano en la eurozona. Esta cumbre podría considerarse como el primer paso hacia el liderazgo de Londres y de los nórdicos en asuntos estratégicos en el Báltico. Mientras tanto, por la deuda

9 George Friedman, "Visegrad: A New European Military Force", Stratfor (Geopolitical Weekly), 17 de mayo de 2011, en www.stratfor.org 
soberana en la eurozona, Berlín está forzando reformas fiscales en este bloque y París lo apoya. Sin embargo, una Europa unificada bajo el liderazgo alemán, históricamente ha sido una preocupación para Londres. Así, este grupo reintroduce a Gran Bretaña como un importante actor en la región. Londres ha afirmado que si el grupo avanza en consolidación, esta cumbre puede ser permanente en sus reuniones, algo que Moscú examinará de cerca. ${ }^{10}$

En la medida en que Europa Oriental actúa para contener el resurgimiento de Rusia en la región, ésta ha respondido con negociaciones sobre seguridad con países de Europa Occidental, una táctica que fortalecía los nexos de Moscú con Europa Occidental (particularmente con Alemania) y hacía que la percepción de los países de Europa Oriental pareciera irrazonable, por lo que la creciente fisura entre Europa Occidental y Europa Oriental podía conducir a una crisis toda vez que ésta trata de evitar ser una zona de amortiguamiento entre Rusia y Washington. Pero el conflicto en Ucrania ha revertido esta situación y ha alineado a Alemania con Estados Unidos y los países del Intermarium. Estos juegos de poder entre las grandes potencias para los realistas sistémicos, como John J. Mearsheimer, se deben a que:

La estructura o arquitectura del sistema internacional obliga a los Estados a buscar el poder, ya que en un sistema mundial en el que no hay una autoridad superior que se encuentre por encima de los grandes poderes, y donde no hay ninguna garantía de que uno no va a atacar a otro, se vuelve lógico para cada estado ser lo suficientemente poderoso como para protegerse en caso de un ataque. En esencia, las grandes potencias se encuentran atrapadas en una jaula de hierro donde no tienen más remedio que competir entre sí por el poder, si quieren sobrevivir. ${ }^{11}$

10 "The Baltic-Nordic-British Relationship Summit", Stratfor, 18 de enero de 2011, en www.stratfor.org

11 John J. Mearsheimer, "Structural Realism”, en Tim Dunne, Milja Kurki y Steve Smith (eds.), International Relations Theories: Discipline and Diversity, $3^{\mathrm{a}}$ ed., Oxford, Oxford University Press, 2013, pp. 77-93. 


\section{EL CONFLICTO EN UCRANIA COMO DETONADOR DE LA CRISIS ENTRE RUSIA Y OCCIDENTE}

Al final de 2013, la Unión Europea (UE) propuso a varios países del Este de Europa, entre éstos a Ucrania, firmar el Acuerdo de Asociación Oriental (AAO), acto que debía tener lugar los días 28 y 29 de noviembre de 2013, en Vilnius. Sin embargo, el presidente Yanukóvich rechazó firmar este acuerdo poco antes del encuentro. La reacción occidental fue de gran descontento. El 21 de noviembre de 2013, el primer ministro Nikolai Azarov declaró que el acuerdo no se firmó por las exigencias del Fondo Monetario Internacional (FMI) de elevar un $40 \%$ el precio del gas para consumo doméstico y de congelar los salarios y pensiones. ${ }^{12}$ Tres partidos de oposición y, sobre todo, el partido Svoboda (Libertad) de Oleg Tyahnybok llevaron al Parlamento, como respuesta a la negativa del gobierno de Yanukovich de firmar el acuerdo con la UE, el proyecto de decreto "Sobre la responsabilidad del gabinete de ministros de Ucrania". ${ }^{13}$

La firma del AAO con la UE significaría un punto de quiebre en las relaciones entre Europa y Rusia y alteraría drásticamente el balance de poder en Europa del Este, ${ }^{14}$ ya que Rusia aún conserva influencia política sobre Ucrania. La estrategia de Ucrania, desde 1992, ha sido maniobrar entre Rusia, uE y Estados Unidos. A pesar de que Yanukóvich provenía del clan de oligarcas de Ucrania del Este, más proclive a Rusia, continuó negociando el acuerdo de asociación con la uE, a causa de la profunda crisis económica del país. Kiev ha tenido que pedir préstamos tanto al FMI, como a Rusia. La

12 Anastasia Kovaleva, “Сколько стоит билет до Опусколова?” (“¿Cuánto cuesta el boleto a Opuskolkova?”), Evrazia, 27 de noviembre de 2013, en http:/ / evrazia.org/search.php?query

13 Valeri Korovin, “Жители Украины должны испытывать ужас перед проектом интеграции с Евросоюзом" ("Los habitantes de Ucrania deben sentir terror ante el proyecto de integración con la UE”), 2 de diciembre de 2013, en http:/ / evrazia.org/article/2421

${ }^{14}$ Clara Weiss, "EU in tug of war with Russia over Ukraine", World Socialist, 28 de noviembre de 2013, en https://www.wsws.org/en/articles/2013/11/08/ukran08.html 
deuda de Ucrania en ese momento era de 60000 millones de dólares. La economía del país creció sólo $0.2 \%$ en 2012 y se contrajo a $-0.5 \%$ en $2013 .{ }^{15}$ La deuda externa bruta para el 1 de enero de 2015 ascendió a 126307 millones de dólares. Si en 2014 la deuda externa superaba los dos tercios del piB del país (71\%), según los pronósticos del Banco Nacional, para 2015 ascendió al 93\%. Según informó el entonces primer ministro Arseni Yatseniuk, Ucrania estaba obligada a pagar casi 11000 millones de dólares de deuda externa en $2015 .{ }^{16}$

El texto del documento del acuerdo de asociación de 1200 páginas estuvo en elaboración siete años y listo para la firma durante un año. El acuerdo consistía en vincular económica y políticamente a Ucrania con la UE, socavando la influencia rusa, y su cancelación es un serio retroceso para el avance de la uE, liderada por Alemania para expandir su influencia al Este, lo que afecta la esfera de influencia de Rusia. Parece una postura contradictoria de Alemania, ya que se había acercado mucho a Rusia, según se ha planteado. Sin embargo, el acercamiento ha sido muy pragmático, porque Rusia la abastece de materias primas, lo que no significa que este país deje de buscar el aumento de su influencia en Europa, aun en detrimento de Rusia. Incluso puede afirmarse que para Alemania, una Rusia menos independiente y armada, sería más deseable para su objetivo geopolítico de convertirse en el líder de Europa occidental. A este propósito, la óptica teórica del realismo sistémico de Mearsheimer y Walt puede ser muy útil para comprender el interés nacional de las grandes potencias, como se anotó arriba. Según John J. Mearsheimer:

No sólo los Estados pueden llegar a preocuparse por el equilibrio de poder, sino que adquieren poderosos incentivos para ganar poder a expensas de los demás. Para empezar, las grandes potencias se

15 Peter Schwarz, "The power struggle in Ukraine and America's strategy for global supremacy”, World Socialist, 23 de diciembre de 2004, en https://www.wsws. org/en/articles/2004/12/ukra-23d.html

16 "La estructura de la deuda externa de Ucrania", Sputnik Mundo, 20 de mayo de 2015, en http://mundo.sputniknews.com/economia/20150520/1037565617. html 
temen entre sí. Hay poca confianza entre ellos. Se preocupan sobre las intenciones de otros estados, en gran parte debido a que sus objetivos son tan difíciles de adivinar. El mayor temor es que otro estado podría tener la capacidad, así como el motivo para atacarlos. Este peligro se ve agravado por el hecho de que los estados operan en un sistema anárquico [...]. Las grandes potencias también entienden que operan en un mundo de auto-ayuda. Tienen que depender de sí mismos para asegurar su supervivencia, ya que otros estados son posibles amenazas y porque no hay autoridad más alta a la que pueden recurrir si son atacados. Esto no significa negar que los estados pueden formar alianzas, que a menudo son útiles para tratar con peligrosos adversarios. En el análisis final, sin embargo, los estados no tienen más remedio que poner sus propios intereses por encima de los intereses de otros estados, así como de la llamada comunidad internacional ${ }^{17}$.

El gobierno ruso buscó disuadir a Yanukóvich de firmar este acuerdo con presiones políticas y económicas. En agosto de 2013, Rusia impuso una prohibición temporal de importación de mercancías ucranianas y el gobierno ruso amenazó con aplicar medidas proteccionistas permanentes y además con endurecer las regulaciones de viaje entre ambos países. Previamente a la negativa de Yanukóvich de firmar el acuerdo con la UE, se sostuvo un encuentro entre éste y el presidente Putin, lo que influyó en la decisión de Yanukóvich de abstenerse de firmar, ya que Rusia ofreció una rebaja del precio del gas en un $30 \%$ y un préstamo por quince mil millones de dólares.

El precio del gas otorgado por Rusia se proponía mediante este encuentro en 268.5 dólares por $1000 \mathrm{~m}^{3}$ de gas natural, comparado con el anterior de 400 dólares: "El acuerdo es al menos temporalmente un revés para Washington y la uE que habían estado presionando agresivamente para expandir su influencia en Ucrania a costa de Moscú". ${ }^{18}$ A este respecto, el presidente Putin

${ }^{17}$ Art. cit., p. 74.

18 Johannes Stern, "Russian offer of aid sharpens international power struggle over Ucrania", World Socialist, 18 de diciembre de 2013, p. 4, en https:/ / www. wsws.org/en/articles/2013/12/18/ukra-d18.html 
aseguró a la oposición ucraniana y a las potencias occidentales que el paquete financiero no significaba un acuerdo final entre Rusia y la ue sobre el ingreso de Ucrania a la Unión Aduanera Euroasiática (UAE): "Ucrania es nuestro socio estratégico y aliado en todos los sentidos de la palabra. Sin embargo, quiero llamar su atención al hecho de que esta ayuda no está atada a condiciones [...]. Quiero calmarlos, no hemos discutido el asunto del acceso de Ucrania a la Unión Económica Aduanera (UAE)". ${ }^{19}$

El anuncio del acuerdo vino a agudizar las tensiones económicas y geopolíticas entre Rusia y las potencias occidentales. El ministro ruso de Defensa Sergei Shoigu confirmó un reporte del diario alemán Bild, en el que se anotaba que Rusia desplegó a mediados de diciembre de 2013 varios misiles balísticos nucleares Iskander en Kaliningrad, en la frontera con Polonia y los Estados Bálticos, para contrarrestar los misiles de Estados Unidos desplegados a lo largo de la frontera con Polonia. ${ }^{20}$

El proyecto de la Asociación Aduanera de Rusia para la UNIÓN EURASIÁTICA

Antes de los actuales acontecimientos, Rusia y Ucrania ya habían llevado a cabo un proceso para la formación de un espacio económico común. Desde 1992, sus gobiernos firmaron un acuerdo de libre comercio en el marco de la Comunidad de Estados Independientes (CEI) y el comercio bilateral se construyó sobre esa base. En el año 2013, este acuerdo bilateral fue sustituido por otro multilateral de libre comercio, también en el marco de la Comunidad de Estados Independientes (CEI). Además, hay que señalar que una prioridad para el tercer periodo presidencial de Vladimir Putin es el intento de crear el Proyecto de la Unión Eurasiática, consistente en una nueva integración en el antiguo espacio soviético.

${ }^{19}$ Ibidem., p. 2.

${ }^{20}$ Stephen Lendman, "The battle for Ucraine", Global Research, 12 de diciembre de 2013, en www.globalresearch.ca/the-battle-for-ukraine/5361273 
El presidente Putin planteó este proyecto siendo aún primer ministro de Rusia en octubre de 2011, en un artículo del periódico Izvestia, como uno de sus grandes planes de gobierno para el futuro. En este documento se plantea que "el mundo ha entrado en una época de turbulencias. Además hay una nueva etapa de cambios tecnológicos y cambia la configuración de los mercados globales". ${ }^{21}$ De aquí se desprende la quinta prioridad que ha establecido el nuevo gobierno ruso: "el fortalecimiento de la posición de Rusia en el mundo, antes que nada por medio de una nueva integración en el espacio eurasiático". ${ }^{22}$

La concreción de este objetivo fue en primer lugar la creación en 2015 del Proyecto de la Unión Económica de Eurasia. En este proyecto se incluyen ya Rusia, Bielorrusia y Kazajstán. Esta estructura intenta ser un espacio de integración económica y política. El objetivo de la integración económica euroasiática es crear condiciones favorables para el crecimiento económico de los países que la integran. El retiro de las tarifas aduanales produce un efecto de crecimiento del intercambio comercial. Por ejemplo, con Kazajstán el comercio ha aumentado considerablemente con Rusia en dos años. Por lo tanto, ésta esperaba que Ucrania participara en este proyecto.

Como se ha anotado ya, la situación económica de Ucrania es crítica y se halla al borde de la moratoria. Por ejemplo, durante la época soviética, la ciudad de Járkov descollaba en industria y ciencia. Por los acontecimientos después de la desintegración de la Unión Soviética, la ciudad rusa de Bélgorod, vecina de Járkov, que durante la época soviética era considerada agraria y prácticamente constituía una fuente de recursos naturales para Járkov, hoy produce artículos industriales per capita cuatro veces más que en esa época soviética. Esto significa que la producción industrial y la calidad de vida han decaído en Járkov y que es difícil encontrar alternativas en el estado en que se encuentra hoy la economía ucraniana.

${ }^{21}$ Vladimir Putin, "A new integration project for Eurasia: The future in the making”, Izvestia, 4 de octubre de 2011, p. 4, en http://premier.gov.ru/eng/even ts/news/16622/

${ }^{22}$ Loc. cit. 
Por tanto, el objetivo de la relación ruso-ucraniana, ya en la etapa postsoviética, era aumentar la cooperación en la esfera de producción de naves espaciales, en la energía atómica y en la producción de aviones. Había proyectos concretos como los AH-124, AH-140 у AH-148, aviones pertenecientes a la línea кв Antonov, que Rusia y Ucrania producían conjuntamente. También estaba el proyecto de construcción de un complejo de producción de naves espaciales y, en cuanto a la cooperación en el sector de energía, la construcción del ciclo de combustible para energía atómica de sus plantas de producción de energía eléctrica. Éstos eran proyectos de miles de millones de dólares, que sustancialmente podrían haber elevado las posibilidades de la economía ucraniana y la habrían hecho diversificarse en la era postsoviética.

Así, con el acceso al poder en Rusia del presidente Putin, en este tercer periodo presidencial de 2012, volvió a darse prioridad a un proyecto de integración entre las extintas repúblicas soviéticas, ya que anteriormente los oligarcas rusos no estaban interesados en el proceso de unificación económica y aduanera. Con todo, el presidente Putin ha logrado separar el estado ruso del poder absoluto de los oligarcas y distanciarlos de la toma de decisiones clave, ya que éstos no están interesados en un espacio común económico con otras repúblicas, porque sus intereses están puestos en el capital internacional occidental.

\section{El CHOQUe DE DOS PROYeCTOS GeOPOLÍticos}

En consecuencia, el conflicto de Ucrania, según el politólogo Rick Rozoff, se recrudece cuando Rusia inicia en la práctica su proyecto de unión aduanera para evitar que Ucrania participe en el Acuerdo de Asociación Oriental, considerado la vía por la cual se pretende integrar a Ucrania en la Organización del Atlántico Norte (OTAN). Los barcos ucranianos ya están activos en dos operaciones navales de la OTAN en curso, que pueden ser permanentes: una, en el Mar Mediterráneo, y otra, en el Golfo Árabe. La primera fue la operación activa Endeavor y la segunda, la operación Ocean Shield, y los Estados Unidos continúan manteniendo los ejercicios 
militares anuales denominados Sea Breeze en el Mar de Crimea, donde también se encuentra la Flota del Mar Negro. El objetivo era intentar sacar a Rusia del Mar Mediterráneo y del Mar Negro. ${ }^{23}$ Sin embargo, la negativa de Ucrania puso en cuestión todo el acuerdo de asociación oriental bajo el cual la ue intenta atraer a cinco países más de la extinta Unión Soviética: Georgia, Azerbaiyán, Armenia, Moldova y Belarús. Rusia, a su vez, está invitando a estos países a la UAE.

El fracaso del acuerdo con Ucrania ocurrió después de que el Parlamento Ucraniano rechazó una ley para liberar a Yulia Timoshenko, ex primera ministra de Ucrania, ya que la ue había puesto como condición su liberación para la firma del acuerdo. Así, pues, el entonces gobierno ucraniano emitió un decreto de cancelación de la firma del acuerdo y señaló la intención de tener una cooperación económica más cercana con los estados de la extinta Unión Soviética y de la Unión Aduanera con Rusia. Además, el decreto pedía una negociación tripartita entre Ucrania, la uE y Rusia sobre las relaciones económicas con Ucrania.

El FMi había acordado otorgar el $20 \%$ de un préstamo de 15 mil millones de dólares a Ucrania y había vinculado el resto del préstamo con drásticas medidas de austeridad para la sociedad, las cuales consistían en congelar salarios y pensiones, elevar el costo del gas y la calefacción en un $40 \%$ y el recorte del gasto público. La UE apoyaba las condiciones del FMI y había demandado mayores reformas estructurales que hoy que se han concretado bajo el gobierno de Petró Paroshenko han conducido a la elevación del desempleo en un país ya castigado por una precaria economía. Por el contrario, Rusia ofreció disminuir el precio del gas y un préstamo de igual monto que el FMI, pero sin las exigencias de éste.

Puede concluirse que las condiciones de la ue y del Fmi fueron las causas principales de la negativa previa de Ucrania, ya que el gobierno temía la consecuencia de un estallido social incontrolable que podía poner en peligro la reelección de Yanukóvich en

23 Rick Rozov, “NATO's Ukranian Target: The Black Sea Fleet”, Global Research, 13 de enero de 2014, en http:/ / www.globalresearch.ca/natos-ukrainian-tar get-the-black-sea.../5364936 
2015. Esta situación se suma a que desde junio de 2013, por la crisis presupuestaria, Ucrania no puede pagar el seguro de desempleo y otras prestaciones sociales a los cientos de miles de desempleados y a los que reciben ayuda gubernamental.

\section{El Acuerdo de Asociación Oriental y SU RePERcusión EN UGRANIA}

Según Sergei Glazyev, economista ruso, lo más importante para Occidente y para un amplio círculo de estructuras influyentes es

impedir la creación de un espacio económico unificado de Rusia donde ingrese Ucrania y separar a ésta de Rusia. Éste es el principal sentido de la AAO para Ucrania, porque además de esto, nada positivo traerá para este país, ya que perderá cerca de 152000 millones de dólares por la creciente importación prevista de productos de la UE por Ucrania. Estas cifras fueron calculadas por expertos de la UE y de Ucrania. ${ }^{24}$

Glazyev considera que Ucrania, desde el punto de vista económico, no recibirá nada hasta el 2020, ya que las empresas ucranianas no podrán cumplir con las obligaciones a las que se comprometieron al firmar el tratado. A este respecto, el entonces primer ministro Nikolai Azarov anunció que la cifra exigida para elevar los estándares de las empresas ucranianas con los estándares industriales de la ue equivalía a una suma aproximada de 150000 millones de euros. Según este acuerdo, Ucrania debe renunciar a su soberanía en el sector comercial, ya que aceptó la obligación de cumplir con los reglamentos tecnológicos europeos que las empresas ucranianas no están en posición de lograr y esto debería hacerlo en un plazo de dos a tres años. Además, Ucrania renuncia a su derecho

${ }^{24}$ Sergei Glazyev, entrevista de los medios al Consejero del presidente de la federación rusa S. Glazyev, Moscú, Rusia, 28 de noviembre de 2013, en http:// tvrain.ru/articles/sovetnik_prezidenta_rf_sergej_glazev_nam_neponjatno_pochemu_janukovich_udarilsja_v_ritoriku_timoshenko-357538/?autoplay=false 
soberano en el sector de regulación interna de los subsidios, las adquisiciones gubernamentales y el mercado energético, es decir que Ucrania pasará a la jurisdicción de la uE, sin tener la posibilidad de influir en la toma de decisiones, ya que se establecen normas y cuotas para las exportaciones de cada país miembro.

Con respecto de la unión aduanera que propone Rusia, a Ucrania se le ofreció la posibilidad de comprar gas a precio preferencial dentro de las tarifas del espacio de la unión aduanera, petróleo sin impuestos y el libre acceso de los productos ucranianos al mercado ruso. Ucrania podría comprar gas a 160 dólares por mil $\mathrm{m}^{3}$, lo que significa que este precio sería tres veces más barato que el actual y no tendría el impuesto de exportación. Además, podría ingresar sus productos alimenticios sin control sanitario-veterinario. También representaría la unificación de reglamentos tecnológicos y la supresión de barreras comerciales.

La suma de mejoras de las condiciones comerciales en el caso de la participación de Ucrania en la unión aduanera de Rusia, según estimación de Glazyev:

Se traduciría en 10 mil millones al año la posibilidad de estabilizar la balanza comercial, la balanza de pagos y empezar a crecer y evitar la moratoria en el nivel en que se encuentra hoy la deuda. También podría tener un desarrollo estable por medio de la cooperación con las fábricas rusas: Rusia tiene más de mil acuerdos de cooperación en el sector automotriz ${ }^{25}$.

\section{Para Glazyev:}

La firma del AAO con la UE impediría estas posibilidades y no le otorgaría al país ningún beneficio, ni la visa ni privilegios específicos, solamente dejaría al país sin una política económica independiente, sin política exterior y tomaría la obligación de participar en los conflictos regionales bajo el liderazgo de la UE. ${ }^{26}$

25 Idem.

${ }^{26} I d$. 
Hay que apuntar que en el texto del AAO está claramente estipulado que éste no le da el derecho a Ucrania de participar en la UE; solamente se estipula que abrirá sus fronteras para el acceso de los productos europeos, que después de un mes de firmado el acuerdo entrarían al país y un $70 \%$ de éstos sin tarifas de importación, incluyendo automóviles usados para estimular la producción en la UE. A su vez, por la baja productividad industrial, Ucrania sólo podría vender trigo y aceite, ya que hoy produce $30 \%$ menos que durante la época soviética, porque para Ucrania la desintegración de la Unión Soviética y la formación de fronteras resultó un proceso de destrucción de sus cadenas productivas. Por tanto, el AAO significaría para Ucrania la pérdida de 4 mil millones de dólares anuales, si cierra la posibilidad de participar en la unión aduanera euroasiática (UAE). Sergei Glazyev afirma además que:

En caso de que Ucrania ingresara a la UAE, de entrada ganaría 10 mil millones de dólares, pues adquiriría el gas con un 30\% menos que el precio en que hoy lo adquiere. Pero nuestros opositores de la uE que reconocen estas cifras afirman que en 2020 vendrán los inversionistas europeos a Ucrania y todo lo solucionarán. El problema es que hoy los inversionistas extranjeros están abandonando Ucrania y esto seguirá sucediendo, independientemente o no de que se firme el AAO. ${ }^{27}$

En cuanto a la integración con Occidente, los empresarios del oeste de Ucrania argumentan que el caso de Polonia es un éxito, pues su economía en siete años creció siete veces, porque tenía el propósito claro de crear todas las condiciones para la entrada a la UE, mencionan créditos europeos baratos que otorgó la asociación con la ue y esta situación es la que los lleva a afirmar que en el corto plazo les iría mejor con Rusia, pero a la larga sería negativa la perspectiva. Sin embargo, cabe señalar que en el caso de Polonia hubo una corrección en el modelo económico que al principio era de libre mercado y después tuvo correcciones de corte neokeynesiano y préstamos que hoy la uE ni está en condiciones ni tiene planeado otorgar a Ucrania. 
El CONFLicto EN UCRANIA: GOLPE AL COMPLEJO DE DEFENSA RUSO

Una consecuencia más del conflicto ucraniano para Rusia es la reducción o la suspensión de interacción en el complejo industrial de defensa de ambos países, ya que uno de los importantes abastecedores para la industria de defensa rusa es Ucrania, en rubros como la producción de partes de helicópteros, servicio de mantenimiento a cohetes intercontinentales y partes de aviones. Alrededor de 400 fábricas del complejo de defensa ruso dependen de partes ucranianas. También hay más de 100 fábricas en Ucrania que producen aviones An-140 y An-148, que participan con el 60\% de la producción de detalles complementarios y partes. Rusia produce el $70 \%$ de los aviones, pero Ucrania posee la tecnología, sin la cual la producción de estos aviones no es posible. ${ }^{28}$ De esta manera, la mayoría de las fábricas de defensa de Ucrania cooperan con Rusia. El nivel de esta cooperación es más del 80\%, mientras que en Europa es sólo de 2.5\%. Por tanto, sin la cooperación de Ucrania en este rubro, la industria de defensa rusa vería afectada su producción militar.

Esto se debe a que después de la desintegración soviética, Ucrania heredó cerca del $50 \%$ del complejo militar industrial común con un estimado total de 3500 fábricas. En el complejo de defensa ucraniano se conservó la producción de alta tecnología a cuenta de encargos múltiples y regulares de las fábricas rusas de defensa, pero la industria militar ucraniana no se renovó prácticamente en todo el periodo postsoviético y hoy presenta un enorme rezago y necesita inversión y esto sólo puede ser si continúa la cooperación con Rusia. Al conservar la interacción en producción de armamento, Ucrania y Rusia podrían competir por los mercados de terceros países. Para lograr este propósito, había todas las condiciones antes del derrocamiento del presidente ucraniano Víctor Yanukóvich, ya que había una serie de

28 I. M. Shepelev, “Анализ санкций против России, определение возможного их влияния на развитие отечественного оборонно-промышленного комплекса и промышленности в целом" (“Análisis de las sanciones contra Rusia, determinación de su efecto en el desarrollo del complejo militar”), Экономика, управление и инвестиции (Economía, Administración e Inversiones), 2014, pp. 1-17. 
proyectos de cooperación de Ucrania y Rusia en el complejo de defensa basados en innovación tecnológica. Éstos consisten en proyectos conjuntos para la fabricación de motores aeronáuticos y la construcción de aviones para civiles, mantenimiento de misiles balísticos intercontinentales y construcción de turbinas a gas para barcos.

Todo esto demuestra la significativa dependencia del complejo de defensa ruso con respecto a agregados y partes de las fábricas ucranianas y también que la dependencia del complejo ucraniano de partes y agregados de Rusia es crucial. Por tanto, el rompimiento de los nexos de producción entre ambos países resultaría catastrófico para el complejo de defensa ucraniano y de colosales gastos para el ruso, que en un mediano plazo será complicado compensar. Para las necesidades de las tropas que manejan misiles de significado estratégico, la sustitución de las partes y agregados ucranianos en los próximos años, se traducirá para la industria rusa en un costo de 20 mil millones de dólares. En la cooperación industrial de defensa, según las fuentes de ambas partes participan alrededor de 1300 fábricas, de las cuales 100 pertenecen a Ucrania y cerca del $70 \%$ de las fábricas de defensa ucranianas dependen crucialmente del abastecimiento de sus socios rusos.

Ucrania, si deseara reorientar su industria armamentista al mercado europeo, no podría, porque se la considera un competidor, y si intenta producir para la OTAN ésta tiene otros estándares de producción, lo que conducirá a la destrucción de las fábricas ucranianas. Así, una ruptura de la cooperación pondría en peligro la seguridad nacional de ambos países. Por esta situación, Rusia ha venido sustituyendo paulatinamente partes y agregados militares provenientes de Ucrania desde 2010 y produciéndolos por sí misma o comprándolos en Occidente. Estados Unidos se ha esforzado en reorientar a Ucrania hacia Occidente, con el propósito de que ingrese a la OTAN, lo que tiene un significado sustancial negativo mayor para la efectividad del desempeño de la industria de defensa rusa, que el de todas las sanciones políticas y económicas de Estados Unidos y la uE impuestas a Rusia en 2014. 
Evaluación GEOPOLÍTICA DEL ACUERdo CON LA UE

Según el politólogo ruso Valery Korovin, la esencia del acuerdo con la UE es un objetivo geopolítico y está dirigido a separar a Ucrania de Rusia, por medio de un cordón sanitario y una zona de inestabilidad que impediría el desarrollo de relaciones económicas estrechas entre la UE y Rusia. La función principal de Ucrania bajo este esquema sería convertirse en una zona de inestabilidad, creando la desestabilización, levantamientos políticos y tensión social. Estos fenómenos favorecerían la estrategia estadounidense de separar a Europa Occidental de Rusia, bajo la lógica tradicional de Mackinder. Ucrania bajo esta perspectiva sólo recibiría mil millones de euros como apoyo financiero para firmar el acuerdo, y la perspectiva es la mayor liberalización de la economía, lo que finalmente acabaría con lo que resta de la política social para la población por medio de una nueva terapia de choque como la que sufrió Rusia después de la desintegración de la Unión Soviética, para incluir a la población ucraniana en un severo contexto de mercado. ${ }^{29}$ Así, en

la estrategia estadounidense, Ucrania entrará al espacio geopolítico de Occidente y su separación de Rusia no le permitirá a ésta dar el salto económico, manteniéndola en Asia, porque sin Ucrania, Rusia será una potencia asiática y no eurasiática y Ucrania se convertirá en una ganancia para Occidente en el gran juego geopolítico mundial. ${ }^{30}$

El gobierno ucraniano pidió a la ue quitar el régimen de visas, otorgar más crédito, la modernización del sistema de transportación de gas. Pero Bruselas no concedió estas peticiones, porque se encuentra en crisis y, por tanto, ahora solamente necesita mercados complementarios para la realización de sus mercancías y para

${ }^{29}$ Valery Korovin, art. cit., p. 3.

30 Irina Kornienko, "Украина: «оранжевая» дезинтеграция" ("Ucrania: desintegración «naranja»"), Evrazia, 26 de enero de 2014, en http://evrazia.org/article/2446 
resolver el problema de la gran desocupación que experimenta. Hay una convergencia de intereses entre la UE y Estados Unidos, ya que al firmar el Acuerdo de Asociación Oriental, Ucrania sería un gran mercado de 47 millones de habitantes para la ue y Estados Unidos podría acercar más su armamento a Rusia, ya que una de las cláusulas del tratado plantea el ingreso de armamento occidental al territorio ucraniano, construyendo aún más cerca el cordón sanitario alrededor de Rusia. Por tanto, Ucrania estaría destinada a ser un mercado para tecnologías peligrosas, como la extracción del gas lutita por medio de la fragmentación hidráulica y un campo de experimentación social por medio de la introducción de las recetas ortodoxas de libre mercado sin coberturas sociales. ${ }^{31}$

Ucrania tiene una enorme importancia para Rusia desde el punto de vista geopolítico. Desde finales del siglo xviri fue una parte vital del imperio zarista y la cuna del nacimiento de los eslavos y su primer estado: la Kiev de Rus en el siglo x de nuestra era. Posteriormente, los rusos se expandieron al este hasta llegar a Siberia oriental, que posee frontera marítima con Japón. Después perteneció a la Unión Soviética en calidad de república federada. Por esa razón, ambos países tenían vínculos económicos estrechos; Rusia era el más grande socio comercial de Ucrania. Por su posición geográfica en el Mar Negro, este país era clave para Rusia, para obtener acceso marítimo, pues ahí se encuentra Crimea y, en ésta, la Flota Rusa del Mar Negro, además de que constituye un acceso al Medio Oriente y al Cáucaso. Por esta situación, después del derrocamiento de Yanukóvich, Rusia decidió apoyar un referendo, en esta península y por cuyo resultado el $97 \%$ de la población votó a favor de la reincorporación a Rusia. Finalmente Rusia dio este paso crucial para su seguridad nacional.

Esto ocurrió porque después de que la uE y la oTAN se extendieron hacia los países de Europa del Este, que estuvieron bajo la influencia rusa después de la Segunda Guerra Mundial, la

31 Veronica Krasninikova, directora del Centro de Investigación en Política Exterior e Iniciativas, participación en el Programa Poediniok, Moscú, 5 de diciembre de 2013, en http://russia.tv/brand/show/brand_id/3963 
pérdida de Ucrania significaría el aislamiento de Rusia con respecto de Europa y su degradación a una potencia regional, como se ha señalado ya. Por tanto, la batalla por Ucrania tiene un carácter explosivo. En los medios de comunicación alemanes es evidente el apoyo al avance alemán hacia el este. Incluso en algunos medios se acusa al presidente Putin de conducir una operación de división de Ucrania, aduciendo que sólo desea la confrontación, pues únicamente fronteras firmes pueden proteger su régimen autoritario.

$\mathrm{Al}$ respecto, en el apartado "Peligros militares y amenazas de guerra a la federación rusa de la doctrina militar de la federación rusa”, actualizada en 2015, el gobierno ruso plantea su propia visión del conflicto, cuando argumenta que las principales amenazas exteriores son: a) el crecimiento del potencial militar de la OTAN y su orientación a funciones globales, realizadas en violación al derecho internacional, el acercamiento de la infraestructura militar de los países miembros de la отAN a las fronteras de la Federación Rusa por medio de la sistemática expansión del bloque; $b$ ) la desestabilización de la situación en determinados países y regiones y la ruptura de la estabilidad global y regional; c) el despliegue y aumento de contingentes militares de países extranjeros (grupo de países) en territorios de países fronterizos con la federación rusa y sus aliados y en aguas adyacentes entre otros objetivos con el de ejercer presión política y militar sobre la federación rusa; $d$ ) creación y despliegue de sistemas estratégicos antimisiles que interfieren con la estabilidad global y desbalancean la correlación de fuerzas en la esfera nuclear con capacidad de un golpe global y también el despliegue de sistemas no nucleares de armas de alta precisión. ${ }^{32}$

32 Военная доктрина Российской Федерации (в редакции от 2015 г.) (“Doctrina militar de la federación rusa, redacción de 2015”), Министерство иностранных дел Российской Федерации (Ministerio de Relaciones Exteriores de la Federación Rusa), 03.III.2015, en http://www.mid.ru/foreign_policy/official_documents/ -/asset_publisher/CptICkB6BZ29/content/id/976907/pop_up?_101_INSTANCE_CptICkB6BZ29_viewMode=print\&_101_INSTANCE_CptICkB6BZ29_qrIn $\operatorname{dex}=1$ 
LA RESPUESTA DE OCGIDENTE A LA REINCORPORACIÓN de Crimea a Rusia

Las sanciones de Occidente y las medidas de Rusia para contrarrestarlas

La serie de sanciones de Estados Unidos y la Unión Europea (UE) exige aumentar más el papel del mercado interno y de la política monetaria para garantizar el desarrollo económico de Rusia. Estas sanciones básicamente consisten en limitar el acceso de las compañías rusas a los mercados financieros en forma de créditos, como se anotó arriba. Por tanto, es necesario activar los mecanismos internos de formación de recursos de dinero para sustituir el financiamiento externo. Esto presupone una significativa corrección de la política crediticia y financiera.

\section{La política crediticia y financiera}

En 2014, los países de la ue y Estados Unidos impusieron sanciones a Rusia a causa de los acontecimientos en Ucrania. Estas sanciones consisten en: a) prohibición de acceso a los mercados de capital en forma de préstamos a largo plazo para las compañías rusas y los bancos, y más si éstas pertenecen al Estado; $b$ ) prohibición de importación de alta tecnología dual; c) prohibición de entrada a sus países a políticos del círculo de Putin, lo cual tiene más bien un carácter político y de desgaste contra la figura del presidente ruso.

Estas sanciones cambian el anterior escenario económico y limitan las posibilidades anteriores de solución de problemas y tareas fundamentales como: garantizar el crecimiento económico constante y mejorar la calidad de vida de la población. Si antes el financiamiento de la economía se llevaba a cabo por recursos financieros externos y mercados exteriores, ahora la principal fuente de recursos financieros, si no es que la única, es el mercado interior. En estas condiciones, la creación de fuentes internas de financiamiento de la economía debe ser prioritaria. Esto presupone una significativa corrección de la política crediticia y financiera. 
Los economistas que asesoran el gobierno de Putin, como Glazyev y Ershov, consideran que una estrategia indispensable para responder a las dificultades de las sanciones es la sustitución de importaciones y el crédito interno por la pequeña y mediana industrias. Por la nueva situación, el Banco Central Ruso acaba de bajar la tasa de interés al crédito de 17 a 12\%33. Sin embargo, para modernizar la planta productiva se requerirían 9 trillones de rublos o el 12\% del PIB, pero hay una tendencia de disminución del PIB que pronostica que en 2015 caerá en $4 \%$. Y si en estas condiciones el BCR disminuye, la masa monetaria entonces creará más dificultades para las empresas que necesitan crédito y propiciará una falta de pagos.

En estas condiciones, es indispensable la transición del modelo especulativo de financiamiento a un modelo orientado a la oferta del crédito para el crecimiento estable y la modernización de la economía. Pero esta propuesta, hasta antes de las sanciones, no había tenido resonancia en los administradores del Banco Central, que siguen observando las recetas del FMI, orientadas a los intereses del capital extranjero. El resultado es la profunda dependencia del mercado exterior, la especialización en materia prima, la degradación del sector de inversión y la caída de la industria de la transformación, más la subordinación del sistema financiero a los intereses del capital extranjero y la transferencia anual de 20000 a 50000 millones de dólares al exterior.

El control de la inflación no debe sacrificar otros objetivos de la política macroeconómica, como la garantía de estabilización del rublo, el crecimiento de la inversión, de la producción y el empleo. Otro gran objetivo de la economía rusa postsanciones es aumentar la venta de gas y petróleo a China, para lo cual ya se construye el ducto Fuerza Siberia. También se plantea la supresión de las limitaciones a la exportación de tecnología militar o dual rusa a China.

${ }^{33}$ M. Ershov, Какая экономическая политика нужна России в условиях санкций? (“¿Qué política económica necesita Rusia en condiciones de las sanciones?”), «Вопросы экономики», Revista Problemas de Economía, núm. 12, 2014, pp. 37-53. 
Estas limitaciones se debían a la política rusa de balance entre Estados Unidos y China, pero en las actuales condiciones pierden sentido. Seguramente será aprobado el contrato para abastecer a China de un avión de combate muy sofisticado, el Su-35c. También puede haber avance en los proyectos que hay desde hace años sobre el traspaso de tecnologías atómicas y aeronáuticas y reactores atómicos para aparatos cósmicos. ${ }^{34}$ Así, se abren nuevas posibilidades para la expansión de nexos económicos con los países que no participan en el régimen de sanciones para la búsqueda de la seguridad alimentaria y tecnológica.

\section{Conclusiones}

Las consecuencias de la crisis de 2008 en la economía rusa no habían sido superadas y a éstas se sumaron las dificultades de la caída del precio del petróleo, su menor exportación por la menor demanda y la subsecuente caída del poder adquisitivo del rublo. Esto ha afectado el poder adquisitivo de la población rusa y también a países como Alemania, que exporta mercancías a Rusia. Los problemas que han provocado las sanciones occidentales de 2014 a la economía rusa representan de un 25 a un $30 \%$ de su problemática actual. Desde su reincorporación a Rusia, Crimea también tiene un bloqueo económico.

Como resultado de las sanciones, las compañías rusas se quedaron sin préstamos a largo plazo, lo que limitó su posibilidad de refinanciación. Surgió la amenaza de no poder pagar las deudas y las compañías pidieron ayuda al gobierno ruso. Sin embargo, las reservas del Banco Central Ruso, el Fondo de Reserva y el Fondo de Bienestar Nacional sólo tenían 450 mil millones de dólares y la deuda externa de las compañías rusas se tasó en 630000 millones de dólares. ${ }^{35}$ Rusia a su vez anunció contrasanciones, pues el 28 de

34 V. Kashin, Поворот на Китай: как компенсировать на Востоке потери от западных санкций ("Giro hacia China: cómo compensar en Oriente las pérdidas por las sanciones occidentales”), Forbes, 2014, pp. 1-4.

${ }^{35}$ M. Ershov, art. cit. 
marzo de 2014, el Ministerio de Relaciones Exteriores declaró que las listas de ciudadanos sancionados de la UE, Estados Unidos y Canadá, se ampliaron con un principio de espejo con respecto de las listas de estos países.

Después de que los sistemas de pago Visa y Mastercard, por orden del Ministerio de Finanzas de Estados Unidos, congelaron las operaciones de tarjeta de crédito de varios bancos rusos, se inició un proceso para crear un sistema propio de pagos nacionales. Rusia también aplicó la contrasanción de prohibir la importación de productos agrícolas, frutas y verduras, materias primas y otros alimentos. También se prohibió importar textiles, ropa y otros artículos de industria ligera.

En cuanto a Occidente, desde el punto de vista geopolítico, para la estrategia estadounidense y la de la Unión Europea (UE), la separación de Ucrania con respecto de Rusia es una de sus prioridades y una estrategia puesta en práctica que lleva veinte años. Esta estrategia ha consistido en impedir que Rusia tenga nuevamente influencia en Ucrania por medio de varias tácticas, desde el financiamiento de grupos de oposición, creación de redes sociales proestadounidenses y la realización de la revolución naranja en 2004, cuando Víctor Yúschenko, un presidente proestadounidense, ascendió al poder. Sin embargo, sus cuatro años de presidencia no mejoraron la situación económica y social del país, pero sí empeoraron las relaciones de Ucrania con Rusia, lo que trajo consigo el alza al precio del gas de parte de Moscú para Kiev.

Según el académico ruso F. A. Kazin, "la ampliación de la OTAN desde los años noventa, se ha llevado a cabo prácticamente ignorando el interés ruso y se ha reflejado negativamente en la situación de Rusia en Europa y a nivel global". ${ }^{36}$ Desde este punto de vista, Ucrania es un elemento central para la defensa a largo plazo y la supervivencia del Estado ruso, y esto no se debe a

36 “Zakat Transitologui ili «sieraia zona» Evropi?” (“¿Ocaso de la transitología o la «zona gris» de Europa?”), The Center of International and Regional Policy, San Petersburgo, Rusia, 2004, en http:/ / www.cirp.ru/oldsite/publications/Kazin/ transition_cont_2.htm 
factores étnicos, aunque Ucrania tiene la más grande comunidad rusa fuera de la federación. Aun antes del periodo soviético, Ucrania estaba integrada al corazón industrial y agrícola de Rusia. Hoy no sólo representa el punto de tránsito para el gas natural ruso a Europa, sino que realmente es el punto de conexión de la infraestructura rusa entre el Oeste y el Este, en lo que respecta a ductos, caminos, líneas férreas y carreteras.

Así, política y militarmente, Rusia, sin Ucrania, no puede fácilmente proyectar poder en el Cáucaso norte, ni sobre Belarús, ya que el río Dniéper, la principal vía de transporte acuático de este país, fluye al sur de Ucrania y es cercana y está bien conectada con Polonia y los Países Bálticos. Esta realidad geográfica significa que si el gobierno de Belarús cambiara su posición a favor de Rusia por otra administración, la posición geopolítica de Minsk se hubiera acercado a Ucrania en el periodo en que Yúschenko era presidente. Por tanto, haciendo una prospectiva a largo plazo, es fácil entender por qué el triunfo de un gobierno prooccidental como el de Yúschenko, y también por qué ahora el de Petro Póroshenko, impactó tanto en Rusia. Ucrania, por lo demás, posee una frontera cercana a ciudades como Volgogrado o Moscú y al puerto de Sebastopol en el Mar Negro, que es el único puerto de agua templada cerca de Rusia.

El diario alemán Suddeutsche Zeitung afirmó que Yanukóvich se abstuvo de firmar el acuerdo con la UE, porque, como resultado de este acuerdo, el declive económico y el desempleo serían mayores, y su reelección en 2015 se vería comprometida. Sin embargo, no sólo fue la presión rusa el factor decisivo. La asociación con la UE conducirá a Ucrania al desastre social, ya que el objetivo del acuerdo es transformar a Ucrania en una plataforma de bajos salarios para las compañías europeas, y las reformas de largo alcance reducirán por años la ya de por sí depauperada calidad de vida de la mayoría de la población. Así, tanto Ucrania como Rusia han perdido a causa de este conflicto, y Rusia, por lo demás, se ha visto inmersa en una difícil situación económica, consecuencia de las sanciones de Occidente. 


\section{BibliogRAFíA}

Военная доктрина Российской Федерации (в редакции от 2015 г.) (“Doctrina militar de la federación rusa, redacción de 2015”), Министерство иностранных дел Российской Федерации (Ministerio de Relaciones Exteriores de la Federación Rusa), 03.III.2015, en http://www.mid. ru/foreign_policy/official_documents/-/asset_publisher/CptICkB6BZ29/content/id/976907/pop_up?_101_INSTANCE_CptICkB6BZ29_viewMode=print\&_101_INSTANCE_CptICkB6BZ29_ qrIndex $=1$

Brzezinski, Zbigniew, "A Geostrategy for Eurasia”, Foreign Affairs, vol. 76, núm. 5, 1997, en https://www.foreignaffairs.com/articles/asia/199709-01/geostrategy-eurasia

— El gran tablero mundial. La supremacía estadounidense y sus imperativos geoestratégicos, trad. Mónica Salomón, Barcelona, Paidós, 1998.

Engdahl, F. William, "Ukraine and a Tectonic Shift in Heartland Power", 22 de marzo de 2010, en //www.engdahl.oilgeopolitics.net/print/ Ukraine $\% 20$ and $\% 20$ a $\% 20$ Tectonic $\% 20$ Shift $\% 20$ in $\% 20$ Heartland\%20Power.pdf

Ershov, M., Какая экономическая политика нужна России в условиях санкций? (“¿Qué política económica necesita Rusia en condiciones de las sanciones?”), «Вопросы экономики», Revista Problemas de Economía, núm. 12, 2014, pp. 37-53.

“Europe: A Shifting battleground, Part 1", Stratfor, 7 de junio de 2011, en www.stratfor.org

Friedman, George, "The political crisis in Ukraine is a battle for the country's soul", Stratfor, 2 de diciembre de 2004, p. 1, en www.stratfor.org

_, "Visegrad: A New European Military Force", Stratfor (Geopolitical Weekly), 17 de mayo de 2011, en www.stratfor.org

, "Ucraina: On the Edge of Empires", Geopolitical Weekly, Stratfor (Geopolitical Weekly), 17 de diciembre de 2013, en www.stratfor.org.

Gainutdinova, L., Эксперты об экономических прогнозах на 2015 год, кризисе и его глубине ("Expertos sobre pronósticos económicos para 2015, la crisis y su profundidad"), 6 de marzo de 2015, en http:// worldcrisis.ru/crisis/1840949

Glazyev, Sergei, entrevista de los medios al Consejero del presidente de la federación rusa S. Glazyev, Moscú, Rusia, 28 de noviembre de 2013, 
en http://tvrain.ru/articles/sovetnik_prezidenta_rf_sergej_glazev_n am_neponjatno_pochemu_janukovich_udarilsja_v_ritoriku_timoshenko-357538/?autoplay=false

Kashin, V., Поворот на Китай: как компенсировать на Востоке потери от западных санкций ("Giro hacia China: cómo compensar en Oriente las pérdidas por las sanciones occidentales”), Forbes, 2014, pp. 1-4.

Kazin, F. A., "Zakat Transitologui ili «sieraia zona» Evropi?” (“¿Ocaso de la transitología o la «zona gris» de Europa?”), The Center of International and Regional Policy, San Petersburgo, Rusia, 2004, en http:// www.cirp.ru/oldsite/publications/Kazin/transition_cont_2.htm

Концепция внешней политики Российской Федерации (Утверждена Президентом Российской Федерации В.В.Путиным 12 февраля 2013 г) (“Concepción de política exterior de la federación rusa, ratificada por el presidente de la federación rusa el 12 de febrero de 2013”), Совет Безопасности Российской Федерации (Consejo de Seguridad de la Federación Rusa), 2013, en http://www.scrf.gov.ru/documents/2/25.html

Korneichuk, В., Локализация хозяйственных рисков, порожденных международными санкциями ("Localización de riesgos económicos originados por las sanciones internacionales"), Política Económica, núm. 6, 2014, pp. 126-134.

Kornienko, Irina, "Украина: «оранжевая» дезинтеграция" (“Ucrania: desintegración «naranja»"), Evrazia, 26 de enero de 2014, en http:// evrazia.org/article/2446

Korovin, Valeri, “Жители Украины должны испытывать ужас перед проектом интеграции с Евросоюзом" ("Los habitantes de Ucrania deben sentir terror ante el proyecto de integración con la UE"), 2 de diciembre de 2013, en http:/ / evrazia.org/article/2421

Kovaleva, Anastasia, “Сколько стоит билет до Опусколова?” (“¿Cuánto cuesta el boleto a Opuskolkova?”), Evrazia, 27 de noviembre de 2013, en http://evrazia.org/search.php?query

Krasninikova, Veronica, directora del Centro de Investigación en Política Exterior e Iniciativas, participación en el Programa Poediniok, Moscú, 5 de diciembre de 2013, en http://russia.tv/brand/show/brand_ $\mathrm{id} / 3963$

"La estructura de la deuda externa de Ucrania", Sputnik Mundo, 20 de 
mayo de 2015, en http:/ / mundo.sputniknews.com/economia/20150 520/1037565617.html

Lazare, Daniel, "Who was Stepan Bandera?", Jacobin, 24 de septiembre de 2015, en https://www.jacobinmag.com/2015/09/stepan-banderanationalist-euromaidan-right-sector/

Lendman, Stephen, "The battle for Ucraine", Global Research, 12 de diciembre de 2013, en www.globalresearch.ca/the-battle-for-ukraine/5361273

Mackinder, H. J., "The Geographical Pivot of History”, en Democratic Ideals and Reality, Washington DC, National Defense University Press, 1996, pp. 175-194.

Mearsheimer, John J., "Structural Realism”, en Tim Dunne, Milja Kurki y Steve Smith (eds.), International Relations Theories: Discipline and Diversity, $3^{\mathrm{a}}$ ed., Oxford, Oxford University Press, 2013, pp. 77-93.

Michaels, Fergus, "Europe's energy crisis sharpens antagonisms with Russia”, World Socialist, 6 de abril de 2006, en https://www.wsws.org/en/ articles/2006/04/ener-a06.html

Перестроится ли экономика России перед лицом западных санкций? (“¿Se reestructurará la economía de Rusia frente a las sanciones?”), 2015, en http:/ / www.ereport.ru/news/1502131445.htm

Putin, Vladimir, "A new integration project for Eurasia: The future in the making”, Izvestia, 4 de octubre de 2011, en http://premier.gov.ru/ eng/events/news/16622/

Rossoliński-Liebe, Grzegorz, Stepan Bandera: The Life and Afterlife of a Ukrainian Nationalist, Fascism, Genocide, and Cult, Berlín, ibidem Press, 2014

Rozov, Rick, "NATO's Ukranian Target: The Black Sea Fleet”, Global Research, 13 de enero de 2014, en http://www.globalresearch.ca/natosukrainian-target-the-black-sea.../5364936

Saavedra Weise, Agustín, "Los 150 años de Sir Halford J. Mackinder", Centro de Estudios Internacionales para el Desarrollo, Buenos Aires, Argentina, 21 de febrero de 2011, en http:/ /www.ceid.edu.ar/biblioteca/2011/agustin_saavedra_weise_los_150_de_sir_halford_j.pdf

Schwarz, Peter, "The power struggle in Ukraine and America's strategy for global supremacy", World Socialist, 23 de diciembre de 2004, en https://www.wsws.org/en/articles/2004/12/ukra-23d.html , "Pro European Union protests mount in Kiev", World Socialist, 3 
de diciembre de 2013, en http://intsse.com/wswspdf/en/articles/2 013/12/03/proe-d03.pdf

Shepelev, I. М., “Анализ санкций против России, определение возможного их влияния на развитие отечественного оборонно-промышленного комплекса и промышленности в целом" (“Análisis de las sanciones contra Rusia, determinación de su efecto en el desarrollo del complejo militar"), Экономика, управление и инвестиции (Economía, Administración e Inversiones), 2014, pp. 1-17.

Stern, Johannes, "Russian offer of aid sharpens international power struggle over Ucrania”, World Socialist, 18 de diciembre de 2013, en https://www.wsws.org/en/articles/2013/12/18/ukra-d18.html

"The Baltic-Nordic-British Relationship Summit", Stratfor, 18 de enero de 2011, en www.stratfor.org

"Ukraine, US: Cheap Democracy; Getting what You Pay For?", Stratfor 6 de abril de 2006, p. 2, en www.stratfor.org

Weiss, Clara, "EU in tug of war with Russia over Ukraine", World Socialist, 28 de noviembre de 2013, en https://www.wsws.org/en/articles/20 13/11/08/ukra-n08.html 